Journal of Applied Pharmaceutical Science Vol. 5 (06), pp. 015-019, June, 2015

Available online at http://www.japsonline.com

DOI: $10.7324 / \mathrm{JAPS} .2015 .50603$

ISSN 2231-3354 (cc) BY-NC-SA

\title{
Effect of critical medium components and culture conditions on antitubercular pigment production from novel Streptomyces sp D25 isolated from Thar desert, Rajasthan
}

\author{
Radhakrishnan Manikkam, Gopikrishnan Venugopal ${ }^{1}$, Balagurunathan Ramasamy², Vanaja Kumar ${ }^{1 *}$ \\ ${ }^{1}$ Centre for Drug Discovery and Development, Sathyabama University, Chennai - 600 119. Tamil Nadu, INDIA \\ ${ }^{2}$ Department of Microbiology, Periyar University, Salem - 636 011. Tamil Nadu, INDIA
}

\begin{tabular}{l} 
ARTICLE INFO \\
\hline Article history: \\
Received on: $06 / 04 / 2015$ \\
Revised on: $22 / 04 / 2015$ \\
Accepted on: $17 / 05 / 2015$ \\
Available online: $27 / 06 / 2015$ \\
\hline Key words: \\
Streptomyces, Thar desert, \\
agar surface fermentation, \\
one-variable-at-a-time, \\
antitubercular.
\end{tabular}

\begin{abstract}
Effect of culture conditions and critical medium components on antitubercular pigment production from novel Streptomyces sp D25 isolated from Thar desert, Rajasthan was investigated. Antitubercular pigment from Streptomyces sp. D25 was produced by Agar Surface Fermentation (ASF) using yeast extract malt extract agar (YEME) as a basal medium. Effect of incubation period, temperature, carbon source, nitrogen source, minerals and sodium chloride concentration was studied by adopting one-variable-at-a-time method. Crude pigment produced under different conditions was extracted solid-liquid extraction method using ethyl acetate. Responses measured include the growth rate, quantity of crude extract and activity against $S$. aureus MTCC96 and $M$. tuberculosis $\mathrm{H} 37 \mathrm{Rv}$ at $100 \mu \mathrm{g}$ concentrations. Growth and pigment production was correlated with the bioactivity. Of the various conditions tested, maximum growth, pigmentation and bioactivity was observed on $6^{\text {th }}$ day of incubation. Of the various medium components tested, $1 \%$ glucose, fructose and malt extract, $\mathrm{pH} 7$ and 9 , temperature $30^{\circ} \mathrm{C}$ and $40^{\circ} \mathrm{C}, 0.1 \% \mathrm{KNo} 3$ and $0-5 \% \mathrm{NaCl}$ was found to influence the growth, bioactive pigment production and antimicrobial bioactivity. Further statistical based optimization is in progress to prove the effect of interaction of the above variables on antitubercular pigment production from Streptomyces sp D25.
\end{abstract}

\section{INTRODUCTION}

Actinomycetes are the economically valuable bacteria which are ubiquitous in nature with the ability to produce novel secondary metabolites including antibiotics (Berdy, 2012). Secondary metabolites from actinobacteria have a long history in the treatment of TB. Many clinically used anti-TB compounds have been reported from actinomycetes with new structure and novel mechanism of action (Butler and Buss, 2006). Since the discovery of streptomycin, the first antibiotic used for anti-TB therapy obtained from Streptomyces griseus, during 1944, numerous anti-TB antibiotics have been reported from various actinobacterial genera. Importantly, promising candidates such as rifamycin, erythromycin, pacidamycin, caprazamycin, capuramycin and thiolactomycin in clinical trials against MDR-TB are also of actinomycete origin (Souza, 2009).

\footnotetext{
* Corresponding Author

Dr. Vanaja Kumar, Centre for Drug Discovery and Development,

Sathyabama University, Chennai - 600 119. Tamil Nadu, India

E-mail:vvanaja_kumar51@yahoo.co.in; Mobile:09444734005
}

Inspired by this excellent track record, a significant effort was made that resulted in the successful isolation of actinobacteria from terrestrial sources for anti-TB screening programs in the past 50 years.

However, the rate of discovery of new compounds from normal terrestrial actinobacteria has decreased whereas the rate of re-isolation of known compounds has increased (Lam, 2006). Undiscovered species inhabiting unique environments with differing environmental constraints have been thought to be resources of novel compounds (Kurtboke, 2010; George et al., 2012; Radhakrishnan et al., 2014a). Actinomycete strain D25 used in this study was isolated from Thar Desert soil, Rajasthan, India using Starch casein nitrate (SCN) agar medium. Strain D25 produced powdery growth with diffusible yellow-orange pigment production on yeast extract malt extract (YEME) agar medium. The crude pigment showed promising activity against multi drug resistant (MDR) and extensively drug resistant (XDR) strains of $M$. tuberculosis and methicillin resistant aureus by disc diffusion method and luciferase reporter phage (LRP) assay, respectively (Radhakrishnan et al., 2014a). 
Moreover, strain D25 produced bioactive yellow pigment when grown only on solid medium but not in liquid medium (Radhakrishnan et al., 2014b). Production of secondary metabolites by microorganisms highly depends on the strains and species of microorganisms and their nutritional and cultural conditions (Wang et al., 2010; Jose et al., 2011). Minor changes in media composition exert a huge impact on quantity and quality of secondary metabolites and general metabolic profile of microorganisms (Greasham, 1983; Wang et al., 2011).

Hence, optimization of culture medium is essential to ensure enhanced production of desired metabolites. Optimization of culture medium is conventionally done by one factor at a time (OFAT) method which is workable as long as the production process is influenced by a less number of variables (Kanmani et al., 2013). The present study reports the effect of culture conditions and critical medium components on antitubercular pigment production from novel Streptomyces sp D25 isolated from Thar desert, Rajasthan.

\section{MATERIALS AND METHODS}

\section{Effect of incubation period}

Hundred microliter spore suspension of Streptomyces $\mathrm{sp}$ D25 was transferred into ten yeast extract malt extract (YEME) agar plates and spreaded using sterile L- rods. All the plates were incubated at $28^{\circ} \mathrm{C}$ for 10 days. For every 24 hours of fermentation, after scrapping the mycelial growth, crude pigment secreted into the agar medium was extracted using $50 \mathrm{ml}$ of methanol for 24 hours. The solvent portion was collected and concentrated using eppendorf concentrator at $40^{\circ} \mathrm{C}$ and quantified (Eccleston et al., 2008).

Antibacterial activity was tested against Staphylococcus aureus MTCC96 by disc diffusion method at $100 \mu \mathrm{g} / \mathrm{disc}$ concentration (Radhakrishnan et al., 2007). Activity was expressed as zone of inhibition in millimeter. Antitubercular activity was tested against Mycobacterium tuberculosis H37Rv by adopting luciferase reporter phage (LRP) assay at $100 \mu \mathrm{g} / \mathrm{ml}$ concentration. Activity was expressed as percentage reduction in relative light unit (RLU). Extracts showing more than $50 \%$ reduction in RLU, when compared with control, was considered as showing antitubercular activity (Radhakrishnan et al., 2010).

\section{Effect of solvents on extraction of antitubercular pigment}

Streptomyces sp D25 was grown on YEME agar plates for 10 days at $28^{\circ} \mathrm{C}$. At the end of fermentation, crude pigment secreted onto the agar medium was extracted by solid liquid extraction using different organic solvents such as n-hexane, dichloromethane, diethyl ether, chloroform, ethyl acetate and methanol at 1:2 ratio. All the solvent extracts were concentrated using rotary evaporator and quantified. Antibacterial and antitubercular activity was tested against $S$. aureus

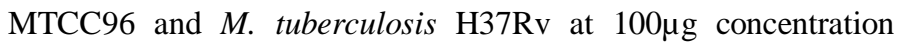
by adopting disc diffusion method and LRP assay, respectively.

\section{Effect of culture conditions and medium components}

Effect of various critical medium components on antitubercular pigment production was studied by adopting classical one-variable-at-a-time method (Shekar et al., 2011). Factors and variables used in this study are given in table 3. Effect of carbons sources were studied by replacing glucose from YEME agar with $1 \%$ of different sugars. Each five plates were prepared for each sugars.

Crude pigment was extracted from different sugar supplemented medium using ethyl acetate after 10 days of incubation at $28^{\circ} \mathrm{C}$. Effect of nitrogen sources were studied by replacing the malt extract with $1 \%$ of different organic and inorganic nitrogen sources. Effect of temperature was studied by incubating twenty five YEME agar plates seeded with $100 \mu \mathrm{L}$ of strain D25 spore suspension. Each five plates were incubated at $10^{\circ} \mathrm{C}, 20^{\circ} \mathrm{C}, 30^{\circ} \mathrm{C}, 40^{\circ} \mathrm{C}$ and $50^{\circ} \mathrm{C}$. After 10 days of incubation, all the plates were taken and extracted using ethyl acetate. Effect of medium $\mathrm{pH}$ on antitubercular pigment production was also tested using YEME agar.

Effect of minerals was studied by supplementing $100 \mathrm{mg}$ of different minerals such as $\mathrm{MgSo} 4, \mathrm{CaCo} 3, \mathrm{KCl}$. Effect of sodium chloride was studied by supplementing different concentration of $\mathrm{NaCl}$ viz., $1 \%, 2.5 \%, 5 \%, 7.5 \%$ and $10 \%$.

\section{Response measurement \\ Growth}

Effect of different variables on the growth of Streptomyces sp D25 was compared with the growth of same strain in YEME agar. Growth pattern was expressed as good (3+), moderate (2+), poor (1+) and no growth (-).

\section{Pigment production}

Quantity of the pigment produced using different variables was estimated using pre-weighed eppendorf tubes and the quantity of crude pigment was expressed in milligrams $/ 50 \mathrm{ml}$ of medium.

Activity

Antibacterial activity was tested against $S$. aureus MTCC96 by disc diffusion method at $100 \mu \mathrm{g} /$ disc concentration (Radhakrishnan et al., 2007). Activity was expressed as zone of inhibition in millimeter. Antitubercular activity was tested against M. tuberculosis H37Rv by adopting LRP assay $100 \mu \mathrm{g} / \mathrm{ml}$ concentration. Activity was expressed in the percentage reduction in relative light unit (RLU). Extracts showing more than $50 \%$ reduction in RLU, when compared with control, was considered as showing antitubercular activity (Radhakrishnan et al., 2010).

\section{RESULTS AND DISCUSSION}

Streptomyces sp D25 produced powdery growth with gray aerial mycelium and soluble yellow pigment on the YEME agar medium. The growth, colour intensity and quantity of 
pigment were increased with incubation period. While testing for bioactivity, crude pigment obtained from $6^{\text {th }}$ day of incubation was showed maximum activity against $S$. aureus MTCC96 and $M$. tuberculosis H37Rv (Table 1).

Table 1: Antituberculous pigment production by strain D25 at different incubation period in agar surface fermentation.

\begin{tabular}{ccccc}
\hline \multicolumn{5}{c}{ Bioactivity against } \\
$\begin{array}{c}\text { Incubation } \\
\text { period } \\
\text { (Days) }\end{array}$ & Growth & $\begin{array}{c}\text { Pigment } \\
\text { production } \\
(\mathbf{m g} / 40 \text { ml) }\end{array}$ & $\begin{array}{c}\text { S. aureus } \\
\text { MTCC96 } \\
\text { (Zone of } \\
\text { inhibition) }\end{array}$ & $\begin{array}{c}\text { M. tuberculosis } \\
\text { H37Rv } \\
\text { (\% red in } \\
\text { RLU) }\end{array}$ \\
\hline $\mathbf{1}$ & - & 0 & NT & NT \\
$\mathbf{2}$ & $1+$ & 7 & 8 & 42.52 \\
$\mathbf{3}$ & $1+$ & 10 & 10 & 51.24 \\
$\mathbf{4}$ & $2+$ & 14 & 12 & 57.49 \\
$\mathbf{5}$ & $2+$ & 30 & 12 & 65.31 \\
$\mathbf{6}$ & $3+$ & 37 & 14 & 80.78 \\
$\mathbf{7}$ & $3+$ & 45 & 14 & 87.78 \\
$\mathbf{8}$ & $3+$ & 48 & 14 & 89.91 \\
$\mathbf{9}$ & $3+$ & 48 & 14 & 89.23 \\
$\mathbf{1 0}$ & $3+$ & 49 & 13 & 85.24 \\
\hline
\end{tabular}

Secondary metabolites are usually produced only at the end of the stationary phase (Sanchez et al., 2010) and secondary metabolism occurs best at sub-maximal growth rates. In many cases, the distinction between the growth phase and production phase is clear (Demain and Fang, 1995). In the present study maximum quantity of pigment production and activity was noticed between the $6^{\text {th }}$ day and $10^{\text {th }}$ day of fermentation. The timing between the two phases can be manipulated by medium optimization in order to improve the product yields.

Among the six different solvents tested for the extraction, yellow pigment got extracted well in all five solvents other than nhexane. In antibacterial activity testing, all the five solvent extracts showed 19-25mm zone of inhibition against $S$. aureus MTCC96. In LRP assay, except n-hexane extract, all other extracts showed more than $80 \%$ reduction in RLU against M. tuberculosis $\mathrm{H} 37 \mathrm{Rv}$ (Table 2).

Table 2: Quantity and activity of crude pigment extracted from strain D25 using different solvents.

\begin{tabular}{lccc}
\hline Extracts & $\begin{array}{c}\text { Quantity of crude } \\
\text { extract } \\
\text { (mg/40 ml of } \\
\text { medium) }\end{array}$ & $\begin{array}{c}\text { S. aureus } \\
\text { MTCC 96 } \\
\text { (zone of } \\
\text { inhibition) }\end{array}$ & $\begin{array}{c}\text { M. tuberculosis } \\
\text { H37Rv } \\
\text { (\% reduction in } \\
\text { RLU) }\end{array}$ \\
\hline ME & 41 & $21.3 \pm 0.57$ & $88.58 \pm 2.31$ \\
ChE & 30 & $23 \pm 1.00$ & $84.74 \pm 3.60$ \\
EAE & 42 & $25 \pm 0.00$ & $90.65 \pm 2.09$ \\
DCME & 35 & $21.3 \pm 0.82$ & $91.59 \pm 4.02$ \\
DEEE & 39 & $19.6 \pm 0.59$ & $91.15 \pm 2.94$ \\
n-HE & - & 0.00 & 0.00 \\
\hline
\end{tabular}

ME - methanol extract; DCME - dichloromethane extract; DEEE - diethyl ether extract; $\mathrm{ChE}$ - chloroform extract; $\mathrm{EAE}$ - ethyl acetate extract; n-HE - nhexane extract; 0 - No zone of inhibition

Most of the secondary metabolites including antibiotics produced by actinomycetes are extracellular in nature (Radhakrishnan et al., 2011). In addition, the results evidenced that the bioactive metabolite present in the crude extract is polar or medium polar in nature. Of the various carbon sources tested, glucose, arabinose, xylose, mannitol, fructose, Inositol and rhamnose were found to influence the growth of strain D25. However, the pigment production and its activity were influenced only by glucose and fructose. Secondary metabolites such as antibiotics are frequently inhibited by a rapidly utilized carbon source such as glucose (Lounes et al., 1996). The basic mechanism(s) of the phenomenon is not understood completely. Glucose is an excellent carbon source for growth, but a high concentration of glucose usually inhibits the biosynthesis of antibiotics (Chu and Li, 2002; Cao, 2003).

All nitrogen sources can be divided into two groups; inorganic nitrogen sources are regarded as quick metabolized nitrogen sources, which are beneficial for fast growth of microorganisms relieving the need of long-time accumulation of product. Moreover, the biosynthesis of antibiotics is also inhibited by rapidly utilized nitrogen sources such as ammonium and regulated by inorganic phosphate (Shikha et al., 2005; Hulya and Tarhan, 2006). Whereas the organic nitrogen sources are sustainable nitrogen sources, which are beneficial for steady product accumulation (Baoxin et al., 2011). In the present study, among the selected organic nitrogen sources tested, growth, pigment production and activity was influenced by the malt extract. Other nitrogen sources like asparagine and glutamine were influenced only the growth of Streptomyces $\mathrm{sp}$ D25.

Actinomycetes have the ability to tolerate wide range of $\mathrm{pH}$ and temperature. Alkaliphilic and halophilic actinomycetes and their antimicrobial and enzymatic potential were reported from desert ecosystems around the world (Hozzein et al., 2004; Norovsuren et al., 2007). Except the $\mathrm{pH} 5$, all other $\mathrm{pH}$ ranges such as 7, 9 and 11 were found to enhance the growth, pigment production and activity. Except $7.5 \%$ and $10 \%$ of $\mathrm{NaCl}$, all other concentrations of $\mathrm{NaCl}$ influenced the growth, pigment production and its bioactivity. Of the various temperature ranges tested, fermentation at $30^{\circ} \mathrm{C}$ and $40^{\circ} \mathrm{C}$ was found to influence the growth, pigment production and activity.

Although many studies of the mineral requirements of microorganisms for growth have been reported, there have been only a few detailed investigations on the requirements of actinomycetes for metals in the biosynthesis of antibiotics (Majumdar and Majumdar, 1965). El-Tayeb et al., (2004) reported that the addition of $\mathrm{KNo} 3$ in the production medium was found to influence the anti TB antibiotic rifamycin production by Amycolatopsis mediterranei Among the different minerals tested, only the KNo3 was found to influence the growth, pigment production and activity.

Good correlation was noted between the growth, pigment production and its activity against $S$. aureus MTCC96 and $M$. tuberculosis $\mathrm{H} 37 \mathrm{Rv}$. Correlation between the quantity of crude pigment and bioactivity was also observed. But there is no correlation between growth and antimicrobial activity and growth and pigment production. 
Table 3: Effect of agar surface fermentation conditions on the antibiotic production by the Streptomyces sp. D25.

\begin{tabular}{|c|c|c|c|c|c|}
\hline \multicolumn{6}{|c|}{ Bioactivity against } \\
\hline Parameters & Variables & Growth & $\begin{array}{l}\text { Quantity of crude } \\
\text { pigment (mg) }\end{array}$ & $\begin{array}{l}\text { S. aureus MTCC96 } \\
\text { (Zone of inhibition) }\end{array}$ & $\begin{array}{l}\text { M. tuberculosis H37Rv } \\
\text { (\% Reduction in RLU) }\end{array}$ \\
\hline \multirow[t]{10}{*}{ Carbon source } & Glucose & $3+$ & 50 & 10 & 80.12 \\
\hline & Arabinose & $3+$ & 0 & 0 & 0.00 \\
\hline & Sucrose & $1+$ & 15 & 7 & 52.45 \\
\hline & Xylose & $3+$ & 15 & 8 & 48.23 \\
\hline & Inositol & $3+$ & 0 & 0 & 0.00 \\
\hline & Mannitol & $3+$ & 0 & 0 & 0.00 \\
\hline & Fructose & $3+$ & 30 & 9 & 83.12 \\
\hline & Rhamnose & $3+$ & 0 & 0 & 0.00 \\
\hline & Raffinose & $1+$ & 0 & 0 & 0.00 \\
\hline & Cellulose & No Growth & 0 & 0 & 0.00 \\
\hline \multirow[t]{6}{*}{ Nitrogen source } & Asparagine & $3+$ & 12 & 7 & 51.00 \\
\hline & Glutamine & $3+$ & 9 & 7 & 49.21 \\
\hline & Tyrosine & $1+$ & 9 & 9 & 45.20 \\
\hline & Malt extract & $3+$ & 47 & 13 & 87.21 \\
\hline & Peptone & $2+$ & 14 & 8 & 60.21 \\
\hline & Beef extract & $2+$ & 11 & 8 & 53.12 \\
\hline \multirow[t]{4}{*}{$\mathrm{pH}$} & 5 & No Growth & 0 & - & 0.00 \\
\hline & 7 & $3+$ & 47 & 12 & 81.22 \\
\hline & 9 & $3+$ & 47 & 10 & 87.24 \\
\hline & 11 & $3+$ & 46 & 10 & 88.24 \\
\hline \multirow[t]{5}{*}{ Temperature } & 10 & No growth & 0 & 0 & 0.00 \\
\hline & 20 & $2+$ & 39 & 11 & 75.24 \\
\hline & 30 & $3+$ & 45 & 12 & 83.12 \\
\hline & 40 & $2+$ & 43 & 9 & 85.44 \\
\hline & 50 & No Growth & 0 & - & 0.00 \\
\hline \multirow[t]{5}{*}{ Minerals } & $\mathrm{MgSo} 4$ & $1+$ & 7 & 7 & 32.21 \\
\hline & KNo3 & $3+$ & 45 & 13 & 83.22 \\
\hline & $\mathrm{KCl}$ & $2+$ & 39 & 11 & 79.24 \\
\hline & $\mathrm{MgCl} 2$ & $2+$ & 36 & 10 & 75.21 \\
\hline & FeSo4 & $1+$ & 8 & 7 & 44.21 \\
\hline \multirow[t]{6}{*}{$\mathrm{NaCl} \%$} & 0 & $3+$ & 48 & 13 & 78.22 \\
\hline & 1 & $3+$ & 51 & 11 & 84.12 \\
\hline & 2.5 & $2+$ & 47 & 13 & 85.39 \\
\hline & 5 & $2+$ & 47 & 14 & 86.24 \\
\hline & 7.5 & $1+$ & 25 & 9 & 66.21 \\
\hline & 10 & No Growth & 0 & - & 0.00 \\
\hline
\end{tabular}

3+ good; $2+$ moderate; $1+$ poor

\section{CONCLUSION}

Findings of the present study as well as our previous studies (Radhakrishnan et al., 2014b) revealed that solid state fermentation is the only choice for the production of antitubercular pigment from the desert soil Streptomyces sp D25. Because the synthetic and purified carbon and nitrogen sources are economically costly, utilizing cheaper substrates like agricultural wastes may be the economically viable alternative for the production of antitubercular pigment from Streptomyces sp D25.

\section{ACKNOWLEDGEMENTS}

The authors thank the Department of Science \& Technology, New Delhi for their support in the form of research grant and National Institute for Research in Tuberculosis, Chennai for the research facilities provided.

\section{REFERENCES}

Baoxin Z, Xiangjing $\mathrm{W}$, Wensheng $\mathrm{Z}$. Optimization of fermentation medium for enhanced production of milbemycin by a mutant of Streptomyces bingchenggensis $\mathrm{BC}-\mathrm{X}-1$ using response surface methodology. African J Biotechnol, 2011; 10(37): 7225-7235.
Berdy J. Thoughts and facts about antibiotics: Where we are now and where we are heading. J. Antibiot, 2012; 65: 385-395

Butler MS, Buss AD. Natural products--the future scaffolds for novel antibiotics?. Biochem Pharmacol, 2006; 71(7):919-929.

Cao FX. The technology of the secondary production and secondary metabolism. National University of Defense Technology Press, Beijing, China, 2003.

Chu J, Li Y. 2002. Studies of modern industrial fermentation control. Chemical Industry Press.

De Souza MV. Promising candidates in clinical trials against multidrug-resistant tuberculosis (MDR-TB) based on natural products. Fitoterapia, 2009; 80: 453-460.

Demain AL, Fang AQ. Emerging Concepts of Secondary Metabolism in Actinomycetes Nippon Hosenkin Gakkaishi, 1995; 9: 98 117.

Eccleston GP, Brooks PR, Kurtboke DI. The occurrence of bioactive Micromonosporae in aquatic habitats of the sunshine coast in Australia. Marine Drugs, 2008; 6: 243-261

El-Tayeb, OM, Hussein MMM, Salama AA, El-Sedawy HF. Optimization of industrial production of rifamycin B by Amycolatopsis mediterranei. II. The role of gene amplification and physiological factors in productivity in shake flasks. African J Biotechnol, 2004; 3(5): 273-280.

George M, Anjumol A, George G, Hatha AAM. Distribution and bioactive potential of soil actinomycetes from different ecological habitats. African J Microbiol Res, 2012; 6(10): 2265-2271.

Greasham RL. 1983. Media for microbial fermentations, in Bioprocessing, Biotechnology, Vol.3, eds Rehm HJ, Read G, Puhler A, Stagler P (New York, NY: VCH Publisher Inc.,128-139. 
Hozzein WN, Li, WJ, Ali MIA, Hammouda O, Mousa AS, Xu, LH, Jiang CL. Nocardiopsis alkaliphila sp. nov., a novel alkaliphilic actinomycete isolated from desert soil in Egypt. Int J Syst Evol Microbiol, 2004; 54: 247-252.

Hulya AK, Tarhan L. Vancomycin antibiotic production and TCA-glyoxalate pathways depending on the glucose concentration in Amycolatopsis orientalis. Enzyme Microb Technol, 2006; 38:727-734.

Jose PA, Santhi VS, Jebakumar SRD. Phylogenetic affiliation, antimicrobial potential and PKS gene sequence analysis of moderately halophilic Streptomyces sp.inhabiting an Indian saltpan. J Basic Microbiol, 2011; 51: 348-356.

Kanmani P, Karthik S, Aravind J, Kumaresan K. The use of response surface methodology as a statistical tool for media optimization in lipase production from the dairy effluent isolate Fusarium solani. ISRN Biotechnol, 2013: 528708.

Kurtboke DI. Biodiscovery from microbial resources: Actinomycetes leading the way. Microbiology Australia, 2010; 31(2): 5357.

Lam KS. Discovery of novel metabolites from marine actinomycetes. Curr Opin Microbiol, 2006; 9: 245-251.

Lounes A, Lebrihi A, Benslimane C. Regulation of spiramycin synthesis in Streptomyces ambofaciens: effects of glucose and inorganic phosphate. Appl Microbiol Biotechnol, 1996; 45:204-211

Majumdar, M. K., and S. K. Majumdar. Effect of minerals on neomycin production by Streptomyces fradiae. Appl Microbiol. 1965;13:190-193.

Norovsuren ZH, Oborotov GV, Zenova GM, Aliev RA, Zvyagintsev DG. Haloalkaliphilic actinomycetes in soils of Mongolian desert steppes. Biological Bulletin, 2007; 34(4):505-507.

Radhakrishnan M, Balagurunathan R, Selvakumar N, Doble M, Vanaja Kumar. Bioprospecting of marine derived actinomycetes with special reference to antimycobacterial activity. Indian J Geo-Mar Sci, 2011; 40(3): 407-410.

Radhakrishnan M, Balaji S, Balagurunathan R. Thermotolerant actinomycetes from Himalayan Mountain-Antagonistic potential, characterization and identification of selected strains. Malaysian Applied Biology, 2007; 36(1): 59-65.

Radhakrishnan Manikkam, Gopikrishnan Venugopal, Balaji Subramaniam, Balagurunathan Ramasamy, and Vanaja Kumar, "Bioactive Potential of Actinomycetes from Less Explored Ecosystems against Mycobacterium tuberculosis and Other Nonmycobacterial Pathogens International Scholarly Research Notices, 2014; Article ID 812974, 9 pages. doi:10.1155/2014/812974
Radhakrishnan M, Pazhanimurugan R, Gopikrishnan V, Balagurunathan R, Vanajakumar. Streptomyces sp D25 isolated from Thar Desert soil, Rajasthan producing pigmented antituberculosis compound only in solid culture. Journal of Pure and Applied Microbiology, 2014b; 8(1): 333-337.

Radhakrishnan M, Suganya S, Balagurunathan R, Vanaja Kumar. Preliminary screening for antibacterial and antimycobacterial activity of actinomycetes from less explored ecosystems. World J Microbiol Biotechnol, 2010; 26: 561-566.

Sánchez S, Chávez A, Forero A, García-Huante Y, Romero A, Sánchez M, Diana Rocha, Sánchez B, Ávalos M, Guzmán-Trampe S, Rodríguez-Sanoja R. Carbon source regulation of antibiotic production. The J Antibiot, 2010; 63: 442-459.

Shekar P, Anuradha J, Radhakrishnan M, Balagurunathan R. Effect of critical medium components on antimicrobial compound production from marine Streptomyces species (A2) by one factor at a time method. Journal of Chemical and Pharmaceutical Research, 2011; 3(6): 872-876.

Shikha D, Rup L, Manjit H. Effect of antibiotics on growth and laccase production from Cyathus bulleri and Pycnoporus cinnabarinus. Bioresour Technol, 2005; 96:1415-1418

Wang X, HuangL, Kang Z, Buchenauer H, Gao X. Optimization of the fermentation process of actinomycete strain Hhs.015T. J Biomedicine Biotechnol, 2010; 141876.doi:10.1155/2010/141876

Wang Y, Fang X, An F, Wang G, Zhang X. Improvement of antibiotic activity of Xenorhabdus bovienii by medium optimization using response surface methodology. Microb Cell Fact, 2011;10:98.

\section{How to cite this article:}

Radhakrishnan M., Venugopal G., Ramasamy B. and Kumar V. Effect of critical medium components and culture conditions on anti-tubercular pigment production from novel Streptomyces sp D25 isolated from Thar desert, Rajasthan. J App Pharm Sci, 2015; 5 (06): 015-019. 